\title{
GEANT4-BASED SIMULATION STUDY OF PEP-II BEAM BACKGROUNDS IN THE BABAR DETECTOR AT THE SLAC B-FACTORY*
}

W. S. Lockman, SCIPP, University of California, Santa Cruz, California 95064, USA

W. Kozanecki, CEA/DSM/DAPNIA, 91991 Gif-sur-Yvette, France

B. Campbell, S. H. Robertson, McGill University, Montreal H3A 2T8, Quebec, Canada

M. Bondioli ${ }^{\dagger}$, G. Calderini, INFN-Pisa, I-56127 Pisa, Italy

N. Barlow, C. L. Edgar, Manchester University, Manchester M13 9PL, United Kingdom

D. Aston, G. Bower, M. Cristinziani, T. Fieguth, D. H. Wright, SLAC, Menlo Park, CA 94025, USA

B. A. Petersen, Stanford University, Stanford, CA 94305, USA

N. L. Blount, D. Strom, University of Oregon, Eugene, OR 97403, USA

\section{Abstract}

To improve the understanding of accelerator-induced backgrounds at the SLAC B-Factory, we simulate lost particle backgrounds in the BABAR detector originating from beam-gas interactions and radiative Bhabha scatters. We have extended the GEANT4-based BABAR detector simulation to include beam-line components and magnetic fields up to $8.5 \mathrm{~m}$ away from the interaction point. We describe the simulation model and then compare preliminary predicted background levels with measurements from dedicated single- and colliding-beam experiments.

\section{INTRODUCTION}

Two potential sources of backgrounds in the BABAR detector [1] include lost particles from beam-gas interactions and Bhabha scatters. The first is single-beam related and scales with the beam current and the residual pressure, while the second is present during colliding beam running only and scales with the luminosity. Two horizontal beamseparation (B1) dipoles (Fig. 1) sweep off-energy particles into the vacuum chamber wall. Secondaries from these interactions are a potential source of machine related background in the BABAR detector. Further improvements in PEP-II luminosity will require raising the beam currents and lowering the $\beta$-function at the interaction point (IP), possibly resulting in increased background levels. To understand the detector backgrounds both in the current and upgraded machine configurations, the detector simulation has been extended to include the machine vacuum chamber, lattice elements and their associated magnetic fields up to and including the final doublet quadrupoles, $\pm 8.5 \mathrm{~m}$ from the IP. We are in the first stages of comparing Monte Carlo predicted detector occupancies with those obtained from single- and colliding beam measurements.

\footnotetext{
* Work supported in part by DOE grant DE-FG02-04ER41286.

$\dagger$ Now at University of California, Irvine, California 92697, USA
}

\section{DETECTOR MONTE CARLO}

\section{Description}

Charged particle tracks in the BABAR detector are measured in a 5-layer silicon vertex tracker (SVT) surrounded by a 40-layer drift chamber (DCH). Both detectors are embedded in a $1.5 \mathrm{~T}$ solenoidal magnetic field. A ringimaging Cherenkov detector (DIRC) surrounds the DCH. Electromagnetic showers from photons and electrons are detected in an array of CsI crystals (EMC) located inside the solenoidal coil of a superconducting magnet.

The detector Monte Carlo program consists of packages for event-generation, particle transport, detector response, and event reconstruction. The Monte Carlo event generators of relevance for this study include the single beam LPTURTLE [2] generator which models beamgas bremsstrahlung and Coulomb scatters, the forward beam-beam bremsstrahlung generator, BBBREM [3] and the small-angle radiative Bhabha generator, BHLUMI [4]. The particle transport package (based on GEANT4 (G4) [5]) is reponsible for tracking charged and neutral particles through the detector and the low energy ring (LER) and high energy ring (HER) beam lines. This package records spatial, temporal and kinematic information ("GHits") in the various subdetectors. The detector response package digitizes the GHit information into raw data ("Digis") and simulates the level-1 trigger. The same reconstruction package is used to process both real and simulated raw data.

The physics processes in the G4-based particle tracking package fall into four categories: decay, electromagnetic, hadronic and electro-nuclear. The decay process is used for all unstable, long-lived particles. The following electromagnetic processes are used: Compton scattering, $\gamma$ conversion and photo-electric effect, multiple scattering, $e^{ \pm}$ionization and bremsstrahlung, $e^{+}$annihilation, $\mu$ ionization and bremsstrahlung, $e^{+}-e^{-}$pair production by 


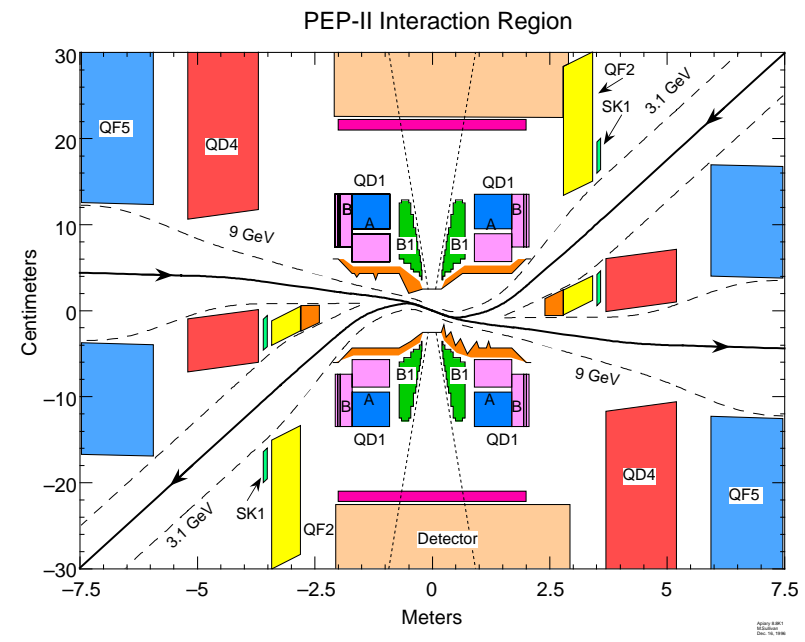

Figure 1: Layout of the PEP-II interaction region, top view. The beam separation dipoles (B1) are shown together with the quadrupoles QD1, QF2, QD4 and QF5. The $9.0 \mathrm{GeV}$ $e^{-}$beam enters from the left, the $3.0 \mathrm{GeV} e^{+}$from the upper right.

muons, and hadron ionization. The hadronic processes include elastic and inelastic scattering, capture and fission. The electro-nuclear processes include $e^{ \pm}$- and $\gamma$-nuclear reactions. A special model is used to treat low energy neutrons from thermal energies up to $20 \mathrm{MeV}$; the Bertini cascade model [6] is used for neutrons above this energy and also for protons and pions. The Low Energy Parameterized model [6] is used for all other hadrons.

To realistically model beam-related backgrounds in the BABAR detector, a detailed model of the vacuum chamber geometry and magnets is required. Unfortunately, the inner surfaces of the vacuum chamber, especially in the region of the QF2 septum, are not easily described by any of the simple constructive solid geometry (CSG) classes available in the G4 toolkit. Instead, we approximate the vacuum chamber using a series of CSG trapezoids which are assembled to form polytubes and polyhedra. The endpoint coordinates of these composite solids are taken from Solid Edge [7] models of the vacuum chamber. The vacuum chamber and magnets as constructed by the G4 simulation are shown in Fig. 2.

\section{Validation}

The geometry of the vacuum chamber is first validated using virtual reality visualization tools to compare each component of the vacuum chamber to its corresponding Solid Edge model. Secondly, aperture locations and shapes derived from transverse slice views at key transition points along the vacuum chamber are compared with the CAD drawings and with the apertures in the LPTURTLE simulation. Finally, from a sample of beam-gas scattered $e^{+}$'s produced by LPTURTLE, we correlate (Fig. 3) the locations in $z$ (the horizontal coordinate in Fig. 1) where par-

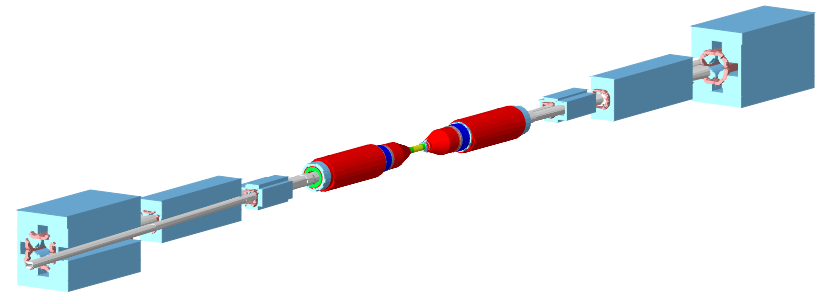

Figure 2: G4 description of PEP-II interaction geometry. The B1 and QD1 magnets appear in red, QF2, QD4 and QF5 magnets in light blue. Part of the LER vacuum chamber (gray) is visible adjacent to QD4 and QF5.

ticles strike the vacuum chamber as predicted by $\mathrm{G} 4$ and LPTURTLE, respectively. This tests that both the vacuum chamber geometry and the beam line optics are consistent between the two simulations. The large peak near $z=200$ $\mathrm{cm}$ is due to a horizontal aperture limitation in the forward LER Q2 septum chamber. Most of the entries in this plot lie along the diagonal, $z_{\mathrm{G} 4}=z_{\mathrm{LPTURTLE}}$.

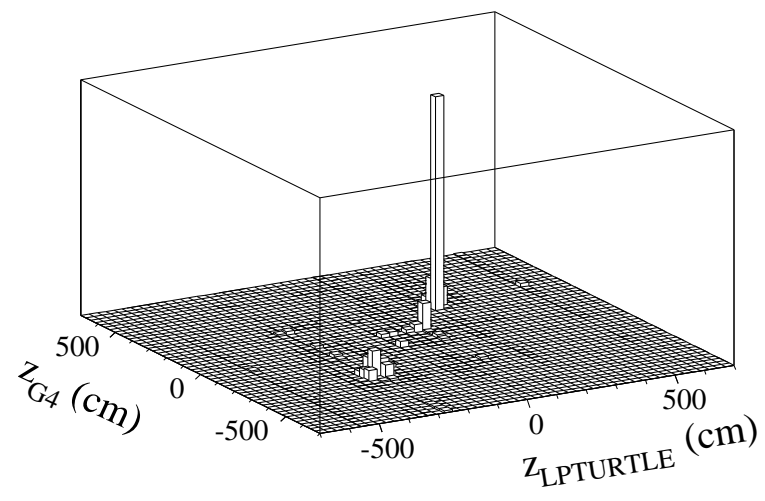

Figure 3: $z$ positions of LER vacuum chamber hits as predicted by the G4 and LPTURTLE simulations, from an ensemble of bremsstrahlung- and Coulomb-scattered $e^{+}$.

\section{Normalization}

In data, an event is defined by all the hits which reside in each subsystem's level-1 (L1) latency buffer when a L1 trigger signal is received. The time window of this buffer, $T$, is subsystem-dependent and is typically of order $1 \mu \mathrm{s}$. In the Monte Carlo, an event is defined as the background hits related to a single Bhabha- or beam-gas scatter. Thus, the occupancy predicted by the simulation must be multiplied by the factor $N_{C}=L \cdot \sigma \cdot T$, where $\sigma$ is the predicted Bhabha scattering cross section, $L$ the instantaneous luminosity and $N_{C}$ the expected number of Bhabha scatters in the time window $T$.

To normalize single beam simulations to the data, we multiply the Monte Carlo occupancy by the factor $N_{S}=$ $I \cdot T \cdot N_{\mathrm{LPTURT}} / t_{\mathrm{LPTURT}}$, where $I$ is the single beam 
current, $N_{\text {LPTURTLE }}$ the number of LPTURTLE events, and $t_{\text {LPTURTLE }}$ the estimated machine time to produce $N_{\text {LPtURTLE }}$ events at a beam current of $1 \mathrm{~A}$ and a $\mathrm{N}_{2}$ equivalent pressure of $1 \mathrm{nTorr}$.

\section{COMPARISON WITH DATA}

The beam-gas and luminosity backgrounds predicted by the simulation described above, have been confronted with systematic measurements of accelerator backgrounds. In dedicated machine experiments, we determined the beamcurrent- and luminosity-dependence of the occupancy in each BABAR subdetector, and parameterized it in terms of beam-gas-, luminosity- and beam-beam-induced backgrounds. The beam-gas terms are measured in singlebeam mode, filling either the LER or the HER. The luminosity term, predicted to be dominated by radiativeBhabha debris, is measured in collision, and is by definition proportional to the measured instantaneous luminosity. The remaining beam-beam term is the difference between the total background-induced occupancy, and the sum of the beam-gas and luminosity terms. Touschek and synchrotron-radiation-induced backgrounds have been shown to be negligible.

The subdetector most sensitive to beam-gas backgrounds is the SVT. It consists of five layers of silicon strip sensors, arranged in overlapping hexagonal patterns around the beam pipe. Each layer is double-sided, with azimuthal $(\phi)$ strips on one face, and axial $(z)$ strips on the other. A comparison of the measured and predicted occupancies in the SVT $\phi$ chips is presented in Fig. 4 for singlebeam backgrounds from the LER. The angle $\phi$ is the azimuthal angle defined in the coordinate system where the $x$-axis is in the PEP-II storage plane pointing outwards and the $y$-axis points vertically upward. The peaks near $\phi=0$ and $\phi=180^{\circ}$ are dominated by degraded-energy positrons swept into the chamber wall by the B1 magnets (Fig. 1). The agreement between data and Monte Carlo, both in shape and overall normalization is remarkably good, considering the simulation assumes a uniform pressure throughout the LER. The other subsystems are in the process of performing similar single-beam comparisons.

To simulate the luminosity backgrounds, we have generated samples of forward beam bremsstrahlung and smallangle radiative Bhabha scattering. However, the results are too preliminary to be presented at this time. In the SVT, DCH and DIRC, the predicted Monte Carlo occupancies from the Bhabha scattering samples appear to be at least one order of magnitude lower than what is observed in data. In the EMC, however, the Monte Carlo occupancies appear to be in better agreement with data, both in magnitude and shape.

The next step in this study will be to model the single beam HER backgrounds. Doing so will provide a better understanding of the systematic uncertainties in the Monte Carlo simulation.
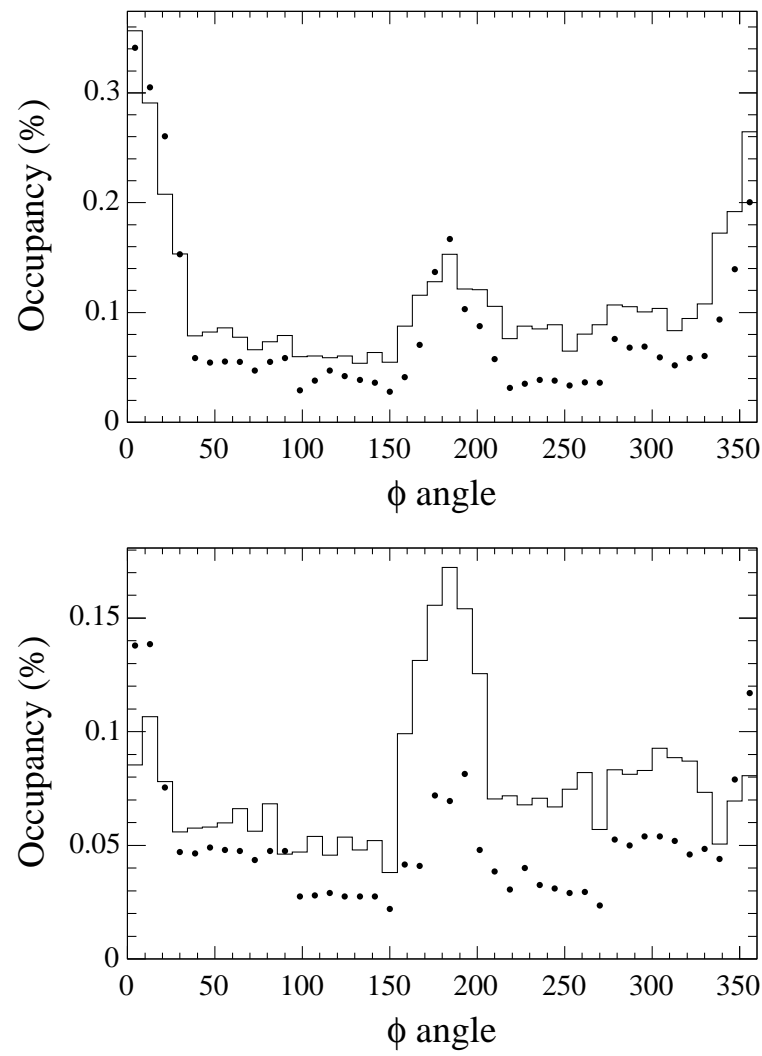

Figure 4: Single-beam SVT $\phi$ chip occupancies measured with a $1 \mathrm{~A} e^{+}$beam (solid circles) and predicted by G4 (histograms) for layer 1 (top) and layer 2 (bottom). The simulation assumes a $1 \mathrm{nTorr}$ pressure around the ring.

\section{REFERENCES}

[1] B. Aubert et al., Nucl. Inst. Meth. A 479, 1 (2002).

[2] T. Fieguth et al.,"Modeling Lost-Particle Backgrounds in PEP-II Using LPTURTLE”, this conference, 1723-TPPP025.

[3] R. Kleiss and H. Burkhardt, Comput. Phys. Commun. 81, 372 (1994).

[4] S. Jadach et al., Comput. Phys. Commun. 102, 229 (1997).

[5] S. Agostinelli et al. [GEANT4 Collaboration], Nucl. Instrum. Meth. A 506, 250 (2003).

[6] See the Geant4 Physics Reference Manual at http://wwwasd.web.cern.ch/wwwasd/geant4/geant4.html (2005).

[7] Solid Edge Computer-Aided Design software, http://www.solidedge.com (2005). 